\title{
Fast Noniterative Steepest Descent Path Algorithm for Planar and Quasi-Planar Patch Geometries
}

LEVENT GüREL*

Dept. of Electrical \& Electr. Eng.

BILKENT UNIVERSITY

Bilkent, Ankara, Turkey

(lgurel@ee.bilkent.edu.tr)

\author{
Weng Cho Chew \\ Ctr. for Computational Electromagnetics \\ DePt. of Electrical \& CoMputer Eng. \\ UNIVERSITY OF ILLINOIS, URBANA, IL 61801 \\ (w-chew@uiuc.edu)
}

\section{Introduction}

Our motivation in developing fast direct (noniterative) algorithms for the solution of planar and quasi-planar geometries is based on the following observations:

- Fast iterative techniques [1-6], which have remarkable performances in solving large problems, are suffering from slow convergence or stagnation for resonant and near-resonant structures, examples of which are shown in Fig. 1.

- Conventional direct solvers, such as the Gaussian elimination, have $O\left(N^{3}\right)$ complexity, which is too high for the solution of large problems.

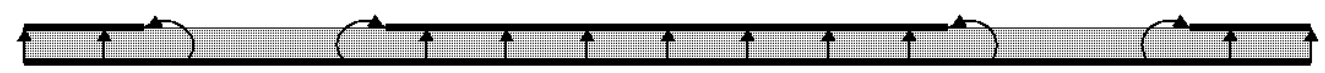

(a)

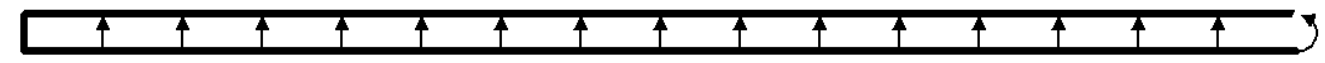

(b)

Figure 1: Examples of resonant structures: (a) planar microstrip geometry, (b) quasi-planar cavity geometry.

Our recent work on recursive T-matrix algorithms (RTMAs) resulted in noniterative solvers with reduced complexities [7-11]. However, the following problems remained open:

- Although RTMAs did reduce the complexity for "dense" geometries, similar reductions were not obtained for $2 \mathrm{D}$ and $3 \mathrm{D}$ planar geometries [11].

- Geometries involving layered media could not be solved using RTMAs.

Both of the above problems are overcome by combining RTMAs with another technique, namely, the steepest descent path (SDP) integration $[12,13]$. 


\section{Steepest Descent Path (SDP) Formulation}

The 3D Green's function (GF) for a homogeneous medium is given by

$$
g\left(\mathbf{r}, \mathbf{r}^{\prime}\right)=\frac{e^{i k\left|\mathbf{r}-\mathbf{r}^{\prime}\right|}}{4 \pi\left|\mathbf{r}-\mathbf{r}^{\prime}\right|}=\frac{i}{8 \pi} \int_{-\infty}^{\infty} d k_{\rho} \frac{k_{\rho}}{k_{z}} H_{0}^{(1)}\left(k_{\rho}\left|\boldsymbol{\rho}-\boldsymbol{\rho}^{\prime}\right|\right) e^{i k_{z}\left|z-z^{\prime}\right|} .
$$

The path of the above integral can be deformed to the SDP and the resulting integral can be discretized to obtain

$$
g\left(\mathbf{r}, \mathbf{r}^{\prime}\right)=\frac{k}{2 \pi} \sum_{m} w_{m} \frac{1+i s_{m}^{2}}{\sqrt{s_{m}^{2}-i 2}} H_{0}^{(1)}\left[k\left|\boldsymbol{\rho}-\boldsymbol{\rho}^{\prime}\right|\left(1+i s_{m}^{2}\right)\right] e^{i k_{z}\left(s_{m}\right)\left|z-z^{\prime}\right|} .
$$

Since the 3D GF can be expressed as a sum of a number of 2D GFs, the 3D problem can be solved by solving the same number of $2 \mathrm{D}$ problems. Note that the equivalent $2 \mathrm{D}$ problems involve "dense" geometries in 2D, making the recursive aggregate-T-matrix algorithm (RATMA) [7] a suitable candidate for a fast 2D solver.

For quasi-planar geometries, $\left|z-z^{\prime}\right|$ remains small for all interactions between pairs of subscatterers, whereas $\left|z-z^{\prime}\right|=0$ for planar geometries. The above formulation can be extended to the layered geometries since the spectral-domain GF is readily available to be used in Eqs. (1) and (2). A symmetrized form of the spectral-domain dyadic GF derived by Chew [14] can be used for this purpose [15]. Special attention should be given to the branch-point and pole contributions when deforming the integration path to the SDP. A similar algorithm for the solution of 2D planar geometries was earlier developed by Chew and Lu $[16,17]$.

RATMA was shown to have a computational complexity of $O\left(N P^{2}\right)[7,11]$ and $P$ was shown to scale as $O(\sqrt{N} \log N)$ for the SDP formulation of 3D planar geometries $[12,13]$. Thus, the overall computational complexity of the fast direct algorithm based on the SDP (FDA/SDP) becomes $O\left(N^{2} \log ^{2} N\right)$, which is significantly lower than $O\left(N^{3}\right)$.

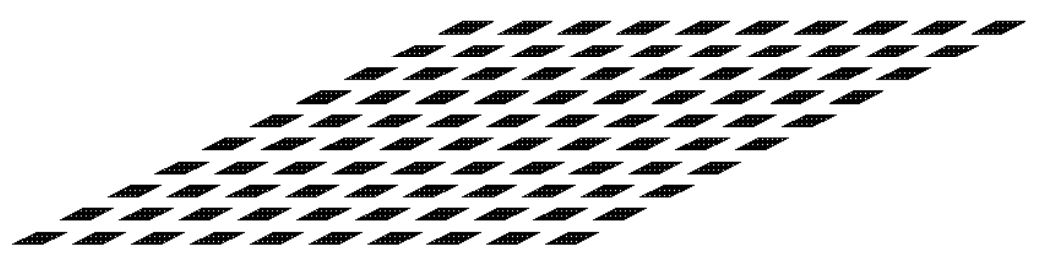

Figure 2: Geometry of a $10 \times 10$ array of 100 conducting square patches with dimensions $k w=1.0$ and spacing $k d=2.0$.

\section{Results}

Figure 2 shows a $10 \times 10$ array of 100 conducting square patches with dimensions $k w=1.0$ and spacing $k d=2.0$. Clearly, FDA/SDP is not limited to and does not 
take advantage of periodic, planar, and canonical geometries involving identical patches. This canonical geometry is chosen merely because of the ease of reproducibility and comparison of the results. Figures 3(a) and (b) show the comparison of TE and TM scattered fields, respectively, as computed by the method of moments (MOM) and FDA/SDP of this paper. For both polarizations, the agreement between the two techniques is very good for the top 20-30 dB of the results. For lower values of RCS, occasional disagreements are observed. This problem is due to the fact that the geometry in Fig. 2 violates the addition theorems of harmonics even though the generalized RATMA [8] is used in this work. This situation can also be remedied by using windowed addition theorems [9]. A better remedy is to use the novel direct algorithms $[18,19]$ that completely bypass this problem.

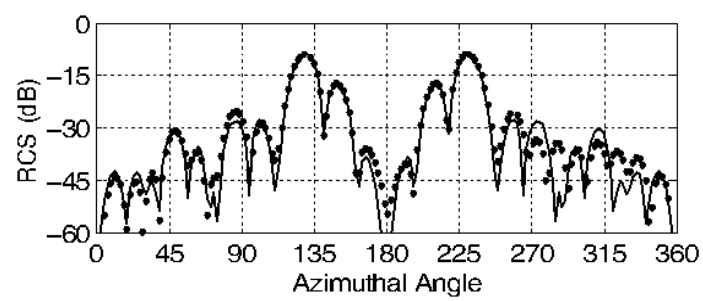

(a)

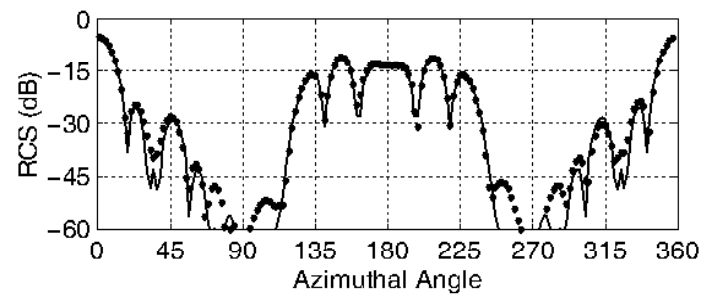

(b)

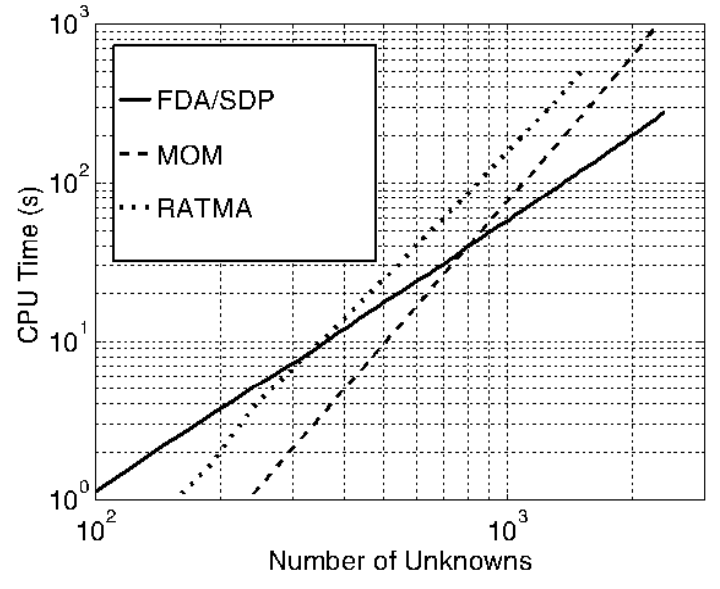

(c)

Figure 3: RCS of the two $\lambda \times \lambda$ conducting patches (as shown in Fig. 2) on the $x-y$ plane for various patch separations: (a) TE and (b) TM polarized RCS curves for the array geometry shown in Fig. 2. Plane waves with TE and TM polarized electric fields are incident on the patches at $\theta=45^{\circ}$ and $\phi=0^{\circ}$. (c) Comparison of the $\mathrm{CPU}$ times required for various noniterative solution algorithms.

Finally, Fig. 3(c) shows a comparison of the solution times of FDA/SDP, MOM, and RATMA. FDA/SDP solution times are seen to increase with a smaller slope than those of MOM and RATMA, confirming the reduced computational complexity of FDA/SDP. The solution times presented in Fig. 3(c) are obtained by solving the scattering problems of increasingly larger planar arrays of patches, as shown in Fig. 2, without taking advantage of the periodicities and the symmetries of these arrays. RATMA was predicted and demonstrated to have $O\left(N^{3}\right)$ complexity for such 2D clustering of 3D scatterers [11]. On the other hand, FDA/SDP employs RATMA to solve 2D problems to take advantage of RATMA's reduced computational complexity for 2D clustering of 2D scatterers [11]. 


\section{References}

[1] V. Rokhlin, "Rapid solution of integral equations of scattering theory in two dimensions," J. Comput. Phys., vol. 86, pp. 414 439, Feb. 1990.

[2] C. C. $\mathrm{Lu}$ and W. C. Chew, "Fast algorithm for solving hybrid integral equations,' Proc. IEE, vol. 140, Part H, pp. 455-460, Dec. 1993.

[3] J. M. Song and W. C. Chew, "Multilevel fast-multipole algorithm for solving combined field integral equations of electromagnetic scattering," Microwave Opt. Tech. Lett., vol. 10, no. 1, pp. 14-19, Sept. 1995.

[4] L. Gürel and M. I. Aksun, "Electromagnetic scattering solution of conducting strips in layered media using the fast multipole method," IEEE Microwave and Guided Wave Lett., vol. 6, no. 8, pp. 277-279, Aug. 1996.

[5] E. Michielssen and W. C. Chew, "Fast steepest descent path algorithm for analyzing scattering from two-dimensional objects," Radio Sci., vol. 31, no. 5, pp. 1215 1224, Sept. Oct. 1996.

[6] E. Bleszynski, M. Bleszynski, and T. Jaroszewicz, "AIM: adaptive integral method for solving large-scale electromagnetic scattering and radiation problems," RS31, no. 5, pp. 122551 , Sept.-Oct. 1996.

[7] W. C. Chew and Y. M. Wang, "A fast algorithm for solution of a scattering problem using a recursive aggregate T matrix method," Microwave Opt. Tech. Lett., vol. 3, no. 5, pp. 164169 , May 1990.

[8] W. C. Chew, L. Gürel, Y. M. Wang, G. Otto, R. Wagner, and Q. H. Liu, "A generalized recursive algorithm for wave-scattering solutions in two dimensions," IEEE Trans. Microwave Theory Tech., vol. MTT-40, pp. 716-723, April 1992.

[9] W. C. Chew, Y. M. Wang, and L. Gürel, "A recursive algorithm using windowed addition theorem," J. of Electromagn. Waves Appl., vol. 6, no. 11, pp. 1537-1560, Nov. 1992.

[10] L. Gürel and W. C. Chew, "A recursive T-matrix algorithm for strips and patches," Radio Science, vol. 27, pp. 387-401, May-June 1992.

[11] L. Gürel and W. C. Chew, "Recursive T-matrix algorithms with reduced complexities for scattering from three-dimensional patch geometries," IEEE Trans. Antennas Propagat., vol. AP-41, pp. 91 99, Jan. 1993.

[12] L. Gürel and W. C. Chew, "Fast direct solution algorithm for electromagnetic scattering from 3D planar and quasi-planar geometries," 1997 IEEE Antennas and Propagation Society International Symposium Digest, Montréal, Canada, pp. 64-67, July 1997.

[13] L. Gürel and W. C. Chew, "Fast algorithm for scattering from planar arrays of conducting patches," IEEE Microwave and Guided Wave Lett., accepted for publication, Nov. 1997.

[14] W. C. Chew, "Symmetrization of the layered-media Green's function," Research Note, Center for Computational Electromagnetics, University of Illinois, May 1996.

[15] J. S. Zhao, W. C. Chew, C. C. Lu, E. Michielssen, and J. M. Song, "Thin-stratified medium fast-multipole algorithm for solving microstrip structures," Research Report No. CCEM-1397, Center for Computational Electromagnetics, University of Illinois, May 1997.

[16] W. C. Chew and C. Lu, "A recursive algorithm to compute the wave-scattering solution of a finite-strip array using an efficient plane-wave basis," Microwave Opt. Tech. Lett., vol. 5, no. 3, pp. 146 148, Mar. 1992.

[17] C. Lu and W. C. Chew, "Electromagnetic scattering of finite strip array on a dielectric slab," IEEE Trans. Microwave Theory Tech., vol. MTT-41, no. 1, pp. 97 100, Jan. 1993.

[18] W. C. Chew and C. C. Lu, "The recursive aggregate interaction matrix algorithm for multiple scatterers," IEEE Trans. Antennas Propagat., vol. AP-43, no. 12, pp. 1483 1486, Dec. 1995.

[19] L. Gürel and W. C. Chew, "Fast direct (noniterative) solvers for integral-equation formulations of scattering problems," 1998 IEEE Antennas and Propagation Society International Symposium Digest, Atlanta, Georgia, June 1998. 